\title{
Synthesis and Antimicrobial Activity of Carboxylate Phosphabetaines Derivatives with Alkyl Chains of Various Lengths
}

\author{
Irina V. Galkina, ${ }^{1}$ Yuliya V. Bakhtiyarova, ${ }^{1}$ Marina P. Shulaeva, ${ }^{2}$ Oskar K. Pozdeev, ${ }^{2}$ \\ Svetlana N. Egorova, ${ }^{3}$ Rafael A. Cherkasov, ${ }^{1}$ and Vladimir I. Galkin ${ }^{1}$ \\ ${ }^{1}$ Department of High Molecular and Organoelement Compounds, A. M. Butlerov Chemistry Institute, Kazan Federal University, \\ Kazan 420008, Russia \\ ${ }^{2}$ Department of Microbiology, Kazan State Medical Academy, Kazan 420012, Russia \\ ${ }^{3}$ Department of Pharmacology, Kazan State Medical University, Kazan 420012, Russia
}

Correspondence should be addressed to Irina V. Galkina; vig54@mail.ru

Received 20 June 2012; Revised 16 August 2012; Accepted 5 September 2012

Academic Editor: Ferenc Billes

Copyright (c) 2013 Irina V. Galkina et al. This is an open access article distributed under the Creative Commons Attribution License, which permits unrestricted use, distribution, and reproduction in any medium, provided the original work is properly cited.

The purpose of the present study was to investigate the antibacterial activity of fifteen novel nanosized alkyl esters of carboxylate phosphabetaine: $\beta$-(carboxyalkyl)ethyltriphenylphosphonium bromides $\quad 4-8, \quad \beta$-(carboxyalkyl)- $\beta$ methylethyltriphenylphosphonium bromides $\mathbf{9 - 1 3}$, and $\beta$-(carboxyalkyl)- $\alpha$-methylethyltriphenylphosphonium bromides 14-18. The in vitro microbiological activity of the synthesized phosphonium bromides against gram-positive and gram-negative bacteria and the yeast Candida albicans was determined in comparison to standard agents. Microbiological results indicate that the synthesized phosphonium salts 4-18 possess a broad spectrum of activity against the tested microorganisms. Every newly synthesized compound was characterized by elemental analyses, IR, ${ }^{1} \mathrm{H}$ NMR, and ${ }^{31} \mathrm{P}$ NMR spectral studies.

\section{Introduction}

Demand for new antimicrobial agents is high because more microorganisms develop resistance against drugs currently available on the market. Resistance of pathogenic bacteria to antibiotics is rapidly becoming a major problem in the medical community and hospital-based healthcare settings. The search for novel agents to combat resistant bacteria has become one of the most important areas of antibacterial research today $[1,2]$. Pharmaceutical and organic chemists are trying to synthesize new drugs with better pharmacokinetic and dynamic properties.

In this study, we prepared triphenyl-substituted phosphonium salts 4-18 on the base of phosphabetaines (1-3) containing alkyl chains of various lengths. The synthesis of such phosphonium salts is very difficult in comparison with ammonium analogs $[3,4]$. In the past years, our group carried out regular research on the synthesis, structure, and reactivity of phosphabetaines of type 1-3, obtained on the basis of tertiary phosphines and unsaturated carboxylic acids [5-8].

The surging interest in this class of compounds becomes quite understandable if we take into account the fact that phosphabetaines are also the original analogs of organic amino acids, with a wide spectrum of potential chemical and biological properties. In these internal phosphonium salts, cationic phosphonium and anionic centers are interconnected not only by ionic but also by covalent bonds. The structure of all products $\mathbf{1 - 3}$ has been confirmed by the direct X-ray method [6-8].

Betaines 1-3 easily react with alkyl halogenides with short alkyl chains to form the corresponding phosphonium salts without biological activity [5-8]. 


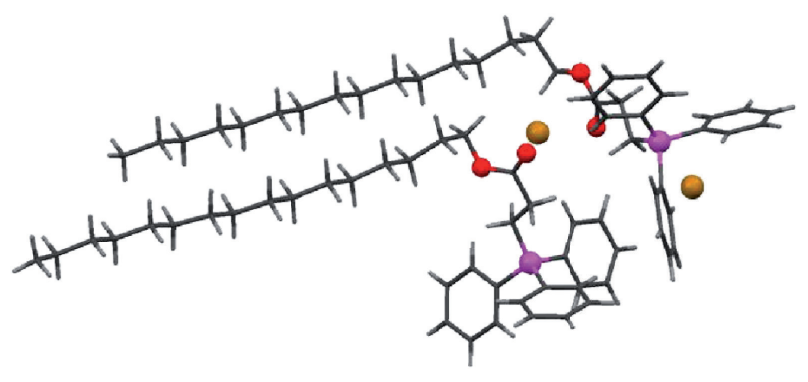

FIGURE 1: Molecular structure of the asymmetric unit of the $\mathrm{Ph}_{3} \mathrm{PCH}_{2} \mathrm{CH}_{2} \mathrm{C}(\mathrm{O}) \mathrm{OC}_{16} \mathrm{H}_{33}$ (7) in crystal.

\section{Experimental}

2.1. Chemistry. All materials were obtained from commercial suppliers and used without purification. Analytical data were obtained from Perkin Elmer 2400 LS and were found within $\pm 0.4 \%$ of the theoretical values. Infrared (IR) spectra were recorded on using $\mathrm{KBr}$ disk on Specord M-80. ${ }^{1} \mathrm{H}$ NMR $\left(\mathrm{D}_{2} \mathrm{O}\right)$ and ${ }^{31} \mathrm{P}$ NMR (DMSO- $\left.\mathrm{d}_{6}\right)$ spectra were determined on a Bruker Avance digital spectrometer $400 \mathrm{MHz}$.

\subsection{Synthesis of $\beta$-(Carboxyalkyl)ethyltriphenylphosphonium Bromides (4-8)}

2.2.1. General Procedure. A mixture of equimolar quantities of $\beta$-triphenylphosphonium ethylcarboxylate 1 (0.01 mol) and appropriate alkyl halogenides $(0.01 \mathrm{~mol})$ was refluxed in dry chloroform $(100 \mathrm{~mL})$ for $2 \mathrm{~h}$. Excess of solvent was removed under reduced pressure. The resulting salts 4-8 were obtained as yellow oils and purified by diethyl ether from starting reagents.

4. Yield (64\%), oil. IR (KBr): $1718(\mathrm{C}=\mathrm{O}), 1135(\mathrm{P}-\mathrm{C})$ $\mathrm{cm}^{-1} ;{ }^{1} \mathrm{H} \mathrm{NMR}\left(\mathrm{D}_{2} \mathrm{O}\right) \delta: 0.75\left(\mathrm{t}, 3 \mathrm{H}, \mathrm{CH}_{3}, J=7.1 \mathrm{~Hz}\right)$, 1.21-1.37 (m, 14H, 7CH $), 2.41-2.56\left(\mathrm{~m}, 2 \mathrm{H}, \mathrm{OCCH}_{2}\right)$, 3.10-3.25 (m, 2H, $\left.\mathrm{PCCH}_{2}\right), 4.11-4.21\left(\mathrm{~m}, 2 \mathrm{H}, \mathrm{PCH}_{2}\right)$, 4.23-4.31 (m, 2H, $\left.\mathrm{OCH}_{2}\right), 7.31-7.54(\mathrm{~m}, 15 \mathrm{H}$, phenyl $\mathrm{H}) ;{ }^{31} \mathrm{P}$ NMR (DMSO-d 6 ) $\delta: 25.58 . \mathrm{C}_{31} \mathrm{H}_{40} \mathrm{BrO}_{2} \mathrm{P}$ (554.90): calcd. C $67.02 \%$, H 7.20\%; found C 66.93\%, H 7.51\%.

5. Yield (71\%), oil. IR (KBr): $1721(\mathrm{C}=\mathrm{O}), 1129(\mathrm{P}-\mathrm{C})$ $\mathrm{cm}^{-1} ;{ }^{1} \mathrm{H} \operatorname{NMR}\left(\mathrm{D}_{2} \mathrm{O}\right) \delta: 0.80\left(\mathrm{t}, 3 \mathrm{H}, \mathrm{CH}_{3}, J=7.0 \mathrm{~Hz}\right)$, 1.25-1.39 (m, $\left.18 \mathrm{H}, 9 \mathrm{CH}_{2}\right), 2.47-2.63\left(\mathrm{~m}, 2 \mathrm{H}, \mathrm{OCCH}_{2}\right)$, 3.11-3.27 (m, 2H, $\left.\mathrm{PCCH}_{2}\right), 4.07-4.19\left(\mathrm{~m}, 2 \mathrm{H}, \mathrm{PCH}_{2}\right)$, 4.28-4.39 (m, 2H, $\left.\mathrm{OCH}_{2}\right), 7.33-7.59(\mathrm{~m}, 15 \mathrm{H}$, phenyl $\mathrm{H}) ;{ }^{31} \mathrm{P}$ NMR (DMSO-d 6 ) $\delta: 25.62 . \mathrm{C}_{33} \mathrm{H}_{44} \mathrm{BrO}_{2} \mathrm{P}$ (582.90): calcd. C $67.92 \%, \mathrm{H} 7.54 \%$; found C $68.03 \%$, $\mathrm{H} 7.87 \%$.

6. Yield (59\%), oil. IR (KBr): $1720(\mathrm{C}=\mathrm{O}), 1130(\mathrm{P}-\mathrm{C})$ $\mathrm{cm}^{-1} ;{ }^{1} \mathrm{H} \operatorname{NMR}\left(\mathrm{D}_{2} \mathrm{O}\right) \delta: 0.81\left(\mathrm{t}, 3 \mathrm{H}, \mathrm{CH}_{3}, J=6.8 \mathrm{~Hz}\right)$, 1.20-1.35 (m, 22H, $\left.11 \mathrm{CH}_{2}\right), 2.43-2.58\left(\mathrm{~m}, 2 \mathrm{H}, \mathrm{OCCH}_{2}\right)$, 3.13-3.29 (m, 2H, $\left.\mathrm{PCCH}_{2}\right), 4.17-4.27\left(\mathrm{~m}, 2 \mathrm{H}, \mathrm{PCH}_{2}\right)$, 4.30-4.37 (m, 2H, $\left.\mathrm{OCH}_{2}\right), 7.30-7.58(\mathrm{~m}, 15 \mathrm{H}$, phenyl $\mathrm{H}) ;{ }^{31} \mathrm{P}$ NMR (DMSO-d ${ }_{6}$ ) $\delta: 25.55 . \mathrm{C}_{35} \mathrm{H}_{48} \mathrm{BrO}_{2} \mathrm{P}$ (610.90): calcd. C $68.74 \%$, H 7.85\%; found C 69.01\%, H, 8.13\%.

7. Yield (65\%), m.p; $208^{\circ} \mathrm{C}$. IR (KBr): 1719 (C=O), 1140 $(\mathrm{P}-\mathrm{C}) \mathrm{cm}^{-1} ;{ }^{1} \mathrm{H}$ NMR $\left(\mathrm{D}_{2} \mathrm{O}\right) \delta: 0.70\left(\mathrm{t}, 3 \mathrm{H}, \mathrm{CH}_{3}, J=\right.$ $6.9 \mathrm{~Hz}), 1.19-1.40\left(\mathrm{~m}, 26 \mathrm{H}, 13 \mathrm{CH}_{2}\right), 2.47-2.63(\mathrm{~m}, 2 \mathrm{H}$, $\left.\mathrm{OCCH}_{2}\right), 3.17-3.27\left(\mathrm{~m}, 2 \mathrm{H}, \mathrm{PCCH}_{2}\right), 4.13-4.29(\mathrm{~m}, 2 \mathrm{H}$,
$\left.\mathrm{PCH}_{2}\right), 4.22-4.39\left(\mathrm{~m}, 2 \mathrm{H}, \mathrm{OCH}_{2}\right), 7.43-7.67$ ( $\mathrm{m}, 15 \mathrm{H}$, phenyl $\mathrm{H}) ;{ }^{31} \mathrm{P}$ NMR (DMSO- $\mathrm{d}_{6}$ ) $\delta: 25.70 . \mathrm{C}_{37} \mathrm{H}_{52} \mathrm{BrO}_{2} \mathrm{P}$ (638.90): calcd. C 69.48\%, H 8.13\%; found C 69.47\%, H 8.47\%.

8. Yield (60\%), oil. IR (KBr): $1720(\mathrm{C}=\mathrm{O}), 1138(\mathrm{P}-\mathrm{C})$ $\mathrm{cm}^{-1} ;{ }^{1} \mathrm{H} \operatorname{NMR}\left(\mathrm{D}_{2} \mathrm{O}\right) \delta: 0.72\left(\mathrm{t}, 3 \mathrm{H}, \mathrm{CH}_{3}, J=6.9 \mathrm{~Hz}\right)$, 1.17-1.27 (m, 30H, 15CH $), 2.47-2.59\left(\mathrm{~m}, 2 \mathrm{H}, \mathrm{OCCH}_{2}\right)$, 3.17-3.36 (m, 2H, $\left.\mathrm{PCCH}_{2}\right), 4.09-4.19\left(\mathrm{~m}, 2 \mathrm{H}, \mathrm{PCH}_{2}\right)$, 4.21-4.37 (m, 2H, $\left.\mathrm{OCH}_{2}\right), 7.38-7.66(\mathrm{~m}, 15 \mathrm{H}$, phenyl $\mathrm{H}) ;{ }^{31} \mathrm{P}$ NMR (DMSO-d ${ }_{6}$ ) $\delta: 25.87 . \mathrm{C}_{39} \mathrm{H}_{56} \mathrm{BrO}_{2} \mathrm{P}$ (666.90): calcd. C $70.16 \%, \mathrm{H} 8.39 \%$; found C $69.89 \%, \mathrm{H} 8.02 \%$.

\subsection{Synthesis of $\beta$-(Carboxyalkyl)- $\beta$-methylethyltriphenyl- phosphonium Bromides (9-13)}

2.3.1. General Procedure. A mixture of equimolar quantities of $\beta$-triphenylphosphonium $\beta$-methylethylcarboxylate 2 $(0.01 \mathrm{~mol})$ and appropriate alkyl halogenides $(0.01 \mathrm{~mol})$ was refluxed in dry chloroform $(100 \mathrm{~mL})$ for $10 \mathrm{~h}$. Excess of solvent was removed under reduced pressure. The resulting salts 9-13 were obtained as yellow oils and purified by diethyl ether from starting reagents.

9. Yield (65\%), oil. IR (KBr): $1710(\mathrm{C}=\mathrm{O}), 1133(\mathrm{P}-\mathrm{C})$ $\mathrm{cm}^{-1} ;{ }^{1} \mathrm{H} \operatorname{NMR}\left(\mathrm{D}_{2} \mathrm{O}\right) \delta: 0.77\left(\mathrm{t}, 3 \mathrm{H}, \mathrm{CH}_{3}, J=7.0 \mathrm{~Hz}\right)$, 0.80-0.95 (m. 3H, $\left.\mathrm{PCCH}_{3}\right), 1.23-1.47\left(\mathrm{~m}, 14 \mathrm{H}, 7 \mathrm{CH}_{2}\right)$, 2.48-2.51 (m, 2H, $\left.\mathrm{OCCH}_{2}\right), 3.10-3.35\left(\mathrm{~m}, 2 \mathrm{H}, \mathrm{PCCH}_{2}\right)$, 4.28-4.39 (m, 2H, $\left.\mathrm{OCH}_{2}\right), 4.69-4.77$ (m. 1H, PCH), 7.27-7.84 (m, 15H, phenyl H); ${ }^{31} \mathrm{P}$ NMR (DMSO-d 6 ) $\delta$ : 24.21. $\mathrm{C}_{31} \mathrm{H}_{42} \mathrm{BrO}_{2} \mathrm{P}$ (556.90): calcd. C 66.79\%, H 7.58\%; found $\mathrm{C} 66.25 \%, \mathrm{H} 7.40 \%$.

10. Yield (70\%), oil. IR (KBr): $1715(\mathrm{C}=\mathrm{O}), 1137$ $(\mathrm{P}-\mathrm{C}) \mathrm{cm}^{-1} ;{ }^{1} \mathrm{H} \operatorname{NMR}\left(\mathrm{D}_{2} \mathrm{O}\right) \delta: 0.80\left(\mathrm{t}, 3 \mathrm{H}, \mathrm{CH}_{3}, J=\right.$ $6.9 \mathrm{~Hz}), 0.79-0.93\left(\mathrm{~m} .3 \mathrm{H}, \mathrm{PCCH}_{3}\right), 1.27-1.53(\mathrm{~m}, 18 \mathrm{H}$, $\left.9 \mathrm{CH}_{2}\right), 2.37-2.49\left(\mathrm{~m}, 2 \mathrm{H}, \mathrm{OCCH}_{2}\right), 3.07-3.37(\mathrm{~m}, 2 \mathrm{H}$, $\left.\mathrm{PCCH}_{2}\right), 4.25-4.40\left(\mathrm{~m}, 2 \mathrm{H}, \mathrm{OCH}_{2}\right), 4.73-4.87$ (m. 1H, PCH), 7.25-7.81 (m, 15H, phenyl H); ${ }^{31} \mathrm{P}$ NMR (DMSO-d 6 ) $\delta$ : 24.17. $\mathrm{C}_{33} \mathrm{H}_{46} \mathrm{BrO}_{2} \mathrm{P}$ (584.90): calcd. C 67.69\%, H 7.86\%; found C $67.91 \%$, H 7.79\%.

11. Yield (63\%), oil. IR (KBr): $1712(\mathrm{C}=\mathrm{O}), 1135$ $(\mathrm{P}-\mathrm{C}) \mathrm{cm}^{-1} ;{ }^{1} \mathrm{H} \operatorname{NMR}\left(\mathrm{D}_{2} \mathrm{O}\right) \delta: 0.81\left(\mathrm{t}, 3 \mathrm{H}, \mathrm{CH}_{3}, J=\right.$ $7.3 \mathrm{~Hz}), 0.83-0.97\left(\mathrm{~m} .3 \mathrm{H}, \mathrm{PCCH}_{3}\right), 1.25-1.43(\mathrm{~m}, 22 \mathrm{H}$, $\left.11 \mathrm{CH}_{2}\right), 2.43-2.59\left(\mathrm{~m}, 2 \mathrm{H}, \mathrm{OCCH}_{2}\right), 3.11-3.31(\mathrm{~m}, 2 \mathrm{H}$, $\left.\mathrm{PCCH}_{2}\right), 4.18-4.29\left(\mathrm{~m}, 2 \mathrm{H}, \mathrm{OCH}_{2}\right), 4.81-4.97$ (m. 1H, PCH), 7.27-7.87 (m, 15H, phenyl H); ${ }^{31} \mathrm{P}$ NMR (DMSO-d 6 ) $\delta$ : 24.07. $\mathrm{C}_{35} \mathrm{H}_{50} \mathrm{BrO}_{2} \mathrm{P}$ (612.90): calcd. C 68.52\%, H 8.16\%; found C $69.16 \%, \mathrm{H}, 8.27 \%$.

12. Yield (71\%), m.p; $208^{\circ} \mathrm{C}$. IR $(\mathrm{KBr}): 1715(\mathrm{C}=\mathrm{O})$, $1137(\mathrm{P}-\mathrm{C}) \mathrm{cm}^{-1} ;{ }^{1} \mathrm{H}$ NMR $\left(\mathrm{D}_{2} \mathrm{O}\right) \delta: 0.73\left(\mathrm{t}, 3 \mathrm{H}, \mathrm{CH}_{3}\right.$, $J=6.9 \mathrm{~Hz}), 0.81-0.93\left(\mathrm{~m} .3 \mathrm{H}, \mathrm{PCCH}_{3}\right), 1.27-1.47(\mathrm{~m}$, $\left.26 \mathrm{H}, 13 \mathrm{CH}_{2}\right), 2.49-2.58\left(\mathrm{~m}, 2 \mathrm{H}, \mathrm{OCCH}_{2}\right), 3.15-3.40(\mathrm{~m}$, $\left.2 \mathrm{H}, \mathrm{PCCH}_{2}\right), 4.18-4.29\left(\mathrm{~m}, 2 \mathrm{H}, \mathrm{OCH}_{2}\right), 4.73-4.86(\mathrm{~m} .1 \mathrm{H}$, $\mathrm{PCH}), 7.31-7.93\left(\mathrm{~m}, 15 \mathrm{H}\right.$, phenyl H); ${ }^{31} \mathrm{P}$ NMR (DMSO-d 6 ) $\delta$ : 23.77. $\mathrm{C}_{37} \mathrm{H}_{54} \mathrm{BrO}_{2} \mathrm{P}$ (640.90): calcd. C 69.27\%, H 8.42\%; found $\mathrm{C} 69.58 \%, \mathrm{H} 8.30 \%$.

13. Yield (67\%), oil. IR (KBr): $1720(\mathrm{C}=\mathrm{O}), 1135$ $(\mathrm{P}-\mathrm{C}) \mathrm{cm}^{-1} ;{ }^{1} \mathrm{H} \operatorname{NMR}\left(\mathrm{D}_{2} \mathrm{O}\right) \delta: 0.72\left(\mathrm{t}, 3 \mathrm{H}, \mathrm{CH}_{3}, J=\right.$ $6.9 \mathrm{~Hz}), 0.76-0.83\left(\mathrm{~m} .3 \mathrm{H}, \mathrm{PCCH}_{3}\right), 1.13-1.59(\mathrm{~m}, 30 \mathrm{H}$, $\left.15 \mathrm{CH}_{2}\right), 2.47-2.57\left(\mathrm{~m}, 2 \mathrm{H}, \mathrm{OCCH}_{2}\right), 3.07-3.25(\mathrm{~m}, 2 \mathrm{H}$, $\left.\mathrm{PCCH}_{2}\right), 4.08-4.19\left(\mathrm{~m}, 2 \mathrm{H}, \mathrm{OCH}_{2}\right), 4.73-4.87$ (m. 1H, PCH), 


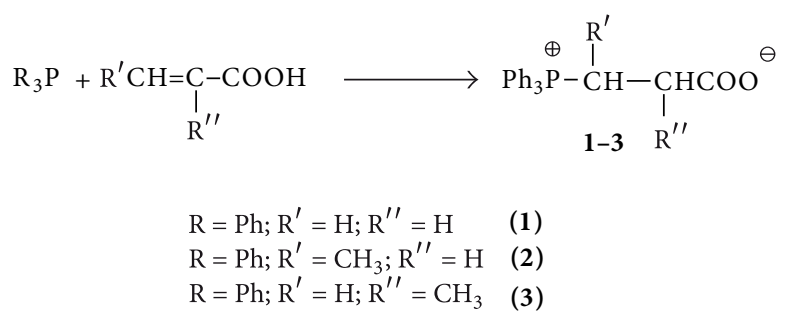

SCHEME 1

\begin{tabular}{|c|c|c|c|c|}
\hline \multirow{7}{*}{$\tilde{\mathrm{N}} \mathrm{H}_{2}=\mathrm{CHCOOH}$} & $\begin{array}{c}\mathrm{Ph}_{3} \mathrm{P}, \mathrm{CHCl}_{3} \\
20^{\circ} \mathrm{C}, 6 \mathrm{~h}\end{array}$ & \multirow{2}{*}{$\underset{\mathrm{Ph}_{3} \mathrm{PCH}_{2} \mathrm{CH}_{2} \mathrm{COO}}{\ominus}$} & $\begin{array}{c}\mathrm{C}_{n} \mathrm{H}_{2 n+1} \mathrm{Br}, \mathrm{CHCl}_{3}, \\
\text { reflux, } 2 \mathrm{~h} \\
\end{array}$ & $\stackrel{\oplus}{\oplus} \ominus$ \\
\hline & & & & $4-8$ \\
\hline & & & & $\mathrm{R}=\mathrm{C}_{10} \mathrm{H}_{21}$ \\
\hline & & & & $\mathrm{R}=\mathrm{C}_{12} \mathrm{H}_{25}$ \\
\hline & & & & $\mathrm{R}=\mathrm{C}_{14} \mathrm{H}_{29}$ \\
\hline & & & & $\mathrm{R}=\mathrm{C}_{16} \mathrm{H}_{33}$ \\
\hline & & & & $\mathrm{R}=\mathrm{C}_{18} \mathrm{H}_{37}(\mathbf{8})$ \\
\hline
\end{tabular}

Scheme 2: Synthetic routes of $\beta$-(carboxyalkyl)ethyltriphenylphosphonium bromides 4-8; reagents and conditions.

7.33-7.89 (m, 15H, phenyl H); ${ }^{31} \mathrm{P}$ NMR (DMSO-d 6 ) $\delta$ : 23.83. $\mathrm{C}_{39} \mathrm{H}_{58} \mathrm{BrO}_{2} \mathrm{P}$ (668.90): calcd. C 69.96\%, H 8.67\%; found C $69.86 \%, \mathrm{H} 8.15 \%$.

\subsection{Synthesis of $\beta$-(Carboxyalkyl)- $\alpha$-methylethyltriphenyl- phosphonium Bromides (14-18)}

2.4.1. General Procedure. A mixture of equimolar quantities of $\beta$-triphenylphosphonium- $\alpha$-methylethylcarboxylate 3 $(0.01 \mathrm{~mol})$ and appropriate alkyl halogenides $(0.01 \mathrm{~mol})$ was refluxed in dry chloroform $(100 \mathrm{~mL})$ for $14 \mathrm{~h}$. Excess of solvent was removed under reduced pressure. The resulting salts 14-18 were obtained as yellow oils and purified by diethyl ether from starting reagents.

14. Yield (73\%), oil. IR (KBr): $1720(\mathrm{C}=\mathrm{O}), 1133(\mathrm{P}-\mathrm{C})$ $\mathrm{cm}^{-1} ;{ }^{1} \mathrm{H}$ NMR $\left(\mathrm{D}_{2} \mathrm{O}\right) \delta: 0.73\left(\mathrm{t}, 3 \mathrm{H}, \mathrm{CH}_{3}, J=7.1 \mathrm{~Hz}\right)$, 1.27-1.43 (m, 14H, 7CH $), 1.57-1.62$ (m. 3H, $\left.\mathrm{PCCCH}_{3}\right)$, 2.47-2.53 (m, 2H, $\left.\mathrm{OCCH}_{2}\right), 3.52-3.59$ (m. 1H, PCCH), 4.15-4.31 (m, 2H, $\left.\mathrm{PCH}_{2}\right), 4.33-4.49\left(\mathrm{~m}, 2 \mathrm{H}, \mathrm{OCH}_{2}\right)$, 7.29-7.52 (m, 15H, phenyl H); ${ }^{31} \mathrm{P}$ NMR $\left(\mathrm{DMSO}^{\left.-\mathrm{d}_{6}\right)} \delta\right.$ : 23.26. $\mathrm{C}_{31} \mathrm{H}_{42} \mathrm{BrO}_{2} \mathrm{P}$ (556.90): calcd. C 66.79\%, H 7.54\%; found C $66.93 \%$, H 7.02\%.

15. Yield (80\%), oil. IR (KBr): $1720(\mathrm{C}=\mathrm{O}), 1130(\mathrm{P}-\mathrm{C})$ $\mathrm{cm}^{-1} ;{ }^{1} \mathrm{H} \operatorname{NMR}\left(\mathrm{D}_{2} \mathrm{O}\right) \delta: 0.71\left(\mathrm{t}, 3 \mathrm{H}, \mathrm{CH}_{3}, J=7.3 \mathrm{~Hz}\right)$, $1.16-1.49\left(\mathrm{~m}, 18 \mathrm{H}, 9 \mathrm{CH}_{2}\right), 1.45-1.67\left(\mathrm{~m} .3 \mathrm{H}, \mathrm{PCCCH}_{3}\right)$, 2.40-2.59 (m, 2H, $\left.\mathrm{OCCH}_{2}\right), 3.45-3.55$ (m. 1H, PCCH), 4.10-4.21 (m, 2H, $\left.\mathrm{PCH}_{2}\right), 4.23-4.51\left(\mathrm{~m}, 2 \mathrm{H}, \mathrm{OCH}_{2}\right)$, 7.16-7.61 (m, 15H, phenyl H); ${ }^{31} \mathrm{P}$ NMR (DMSO-d 6 ) $\delta$ : 23.48. $\mathrm{C}_{33} \mathrm{H}_{46} \mathrm{BrO}_{2} \mathrm{P}$ (584.90): calcd. C 67.69\%, H 7.86\%; found $\mathrm{C} 67.84 \%, \mathrm{H} 7.63 \%$.

16. Yield (77\%), oil. IR (KBr): $1715(\mathrm{C}=\mathrm{O}), 1132(\mathrm{P}-\mathrm{C})$ $\mathrm{cm}^{-1} ;{ }^{1} \mathrm{H} \operatorname{NMR}\left(\mathrm{D}_{2} \mathrm{O}\right) \delta: 0.75\left(\mathrm{t}, 3 \mathrm{H}, \mathrm{CH}_{3}, J=7.1 \mathrm{~Hz}\right)$, $1.31-1.41\left(\mathrm{~m}, 22 \mathrm{H}, 11 \mathrm{CH}_{2}\right), 1.47-1.66\left(\mathrm{~m} .3 \mathrm{H}, \mathrm{PCCCH}_{3}\right)$, 2.43-2.51 (m, 2H, $\left.\mathrm{OCCH}_{2}\right), 3.57-3.66$ (m. 1H, PCCH),
4.27-4.39 (m, 2H, $\left.\mathrm{OCH}_{2}\right), 4.41-4.53\left(\mathrm{~m}, 2 \mathrm{H}, \mathrm{PCH}_{2}\right)$, 7.30-7.63 (m, 15H, phenyl H); ${ }^{31} \mathrm{P}$ NMR (DMSO-d 6 ) $\delta$ : 23.54. $\mathrm{C}_{35} \mathrm{H}_{50} \mathrm{BrO}_{2} \mathrm{P}$ (612.90): calcd. C 68.52\%, $\mathrm{H} 8.16 \%$; found $\mathrm{C} 68.77 \%, \mathrm{H}, 7.05 \%$.

17. Yield (90\%), m.p; $208^{\circ}$ C. IR (KBr): $1720(\mathrm{C}=\mathrm{O})$, $1137(\mathrm{P}-\mathrm{C}) \mathrm{cm}^{-1} ;{ }^{1} \mathrm{H}$ NMR $\left(\mathrm{D}_{2} \mathrm{O}\right) \delta: 0.70\left(\mathrm{t}, 3 \mathrm{H}, \mathrm{CH}_{3}\right.$, $J=7.2 \mathrm{~Hz}), 1.31-1.49\left(\mathrm{~m}, 26 \mathrm{H}, 13 \mathrm{CH}_{2}\right), 1.57-1.72(\mathrm{~m}$. $\left.3 \mathrm{H}, \mathrm{PCCCH}_{3}\right), 2.45-2.56\left(\mathrm{~m}, 2 \mathrm{H}, \mathrm{OCCH}_{2}\right), 3.52-3.59(\mathrm{~m}$. $1 \mathrm{H}, \mathrm{PCCH}), 4.16-4.30\left(\mathrm{~m}, 2 \mathrm{H}, \mathrm{PCH}_{2}\right), 4.33-4.49(\mathrm{~m}, 2 \mathrm{H}$, $\left.\mathrm{OCH}_{2}\right), 7.29-7.52\left(\mathrm{~m}, 15 \mathrm{H}\right.$, phenyl H); ${ }^{31} \mathrm{P} \mathrm{NMR}\left(\mathrm{DMSO}-\mathrm{d}_{6}\right)$ $\delta$ : 23.58. $\mathrm{C}_{37} \mathrm{H}_{54} \mathrm{BrO}_{2} \mathrm{P}$ (640.90): calcd. C 69.27\%, H 8.42\%; found C $68.95 \%, \mathrm{H} 8.11 \%$.

18. Yield (81\%), oil. IR (KBr): $1720(\mathrm{C}=\mathrm{O}), 1133(\mathrm{P}-\mathrm{C})$ $\mathrm{cm}^{-1} ;{ }^{1} \mathrm{H} \operatorname{NMR}\left(\mathrm{D}_{2} \mathrm{O}\right) \delta: 0.73\left(\mathrm{t}, 3 \mathrm{H}, \mathrm{CH}_{3}, J=7.0 \mathrm{~Hz}\right)$, 1.31-1.47 (m, 30H, 15CH $\mathrm{CH}_{2}, 1.62-1.73\left(\mathrm{~m} .3 \mathrm{H}, \mathrm{PCCCH}_{3}\right)$, 2.51-2.63 (m, 2H, $\left.\mathrm{OCCH}_{2}\right), 3.49-3.57$ (m. 1H, PCCH), 4.14-4.29 (m, 2H, $\left.\mathrm{PCH}_{2}\right), 4.31-4.41\left(\mathrm{~m}, 2 \mathrm{H}, \mathrm{OCH}_{2}\right)$, 7.37-7.59 (m, 15H, phenyl H); ${ }^{31} \mathrm{P}$ NMR (DMSO-d 6 ) $\delta$ : 23.60. $\mathrm{C}_{39} \mathrm{H}_{58} \mathrm{BrO}_{2} \mathrm{P}$ (668.90): calcd. C 69.96\%, H 8.67\%; found C $70.24 \%$, H $8.32 \%$.

2.5. Antimicrobial Screening. The antimicrobial activity of the newly synthesized compounds was determined in vitro using the agar disk-diffusion method using Mueller-Hilton agar medium $[9,10]$ against a variety of pathogenic microorganisms: Staphylococcus aureus (ATCC 29213) (Gram-positive bacteria), Escherichia coli (ATCC 25922), Pseudomonas aeruginosa (ATCC 27853) and Proteus mirabilis (ATCC 12453) (Gram-negative bacteria) and fungus Candida albicans (ATCC 885-653). The inhibition zones of the tested compounds were measured after $24-48 \mathrm{~h}$ incubation at $37^{\circ} \mathrm{C}$ for bacteria and after 5 days of incubation at $28^{\circ} \mathrm{C}$ for fungi. Penicillin (Sigma-Aldrich) and Chlorhexidine (SigmaAldrich) were used as reference drug for bacteria, whereas 


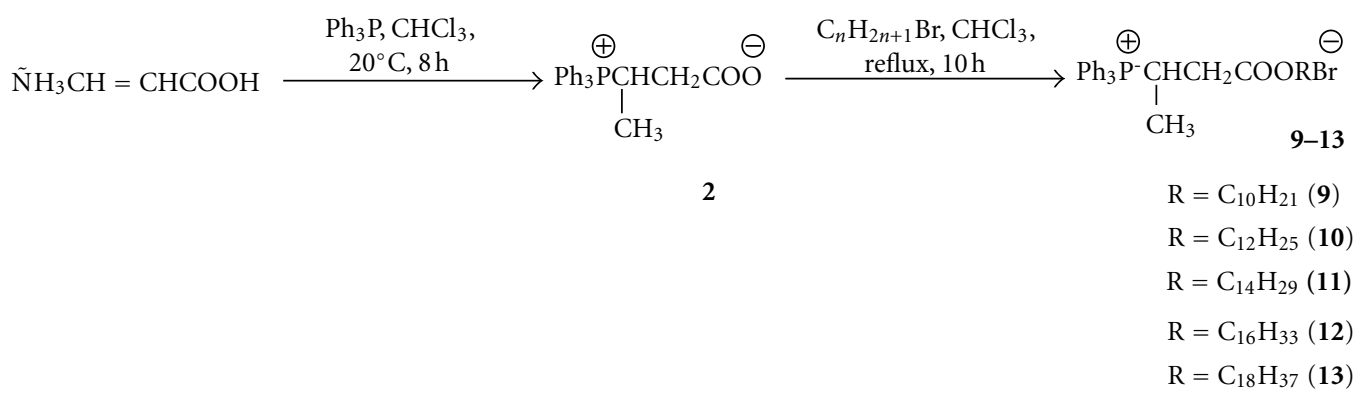

SCHEME 3: Synthetic routes of $\beta$-(carboxyalkyl)- $\beta$-methylethyltriphenylphosphonium bromides 9-13; reagents and conditions.

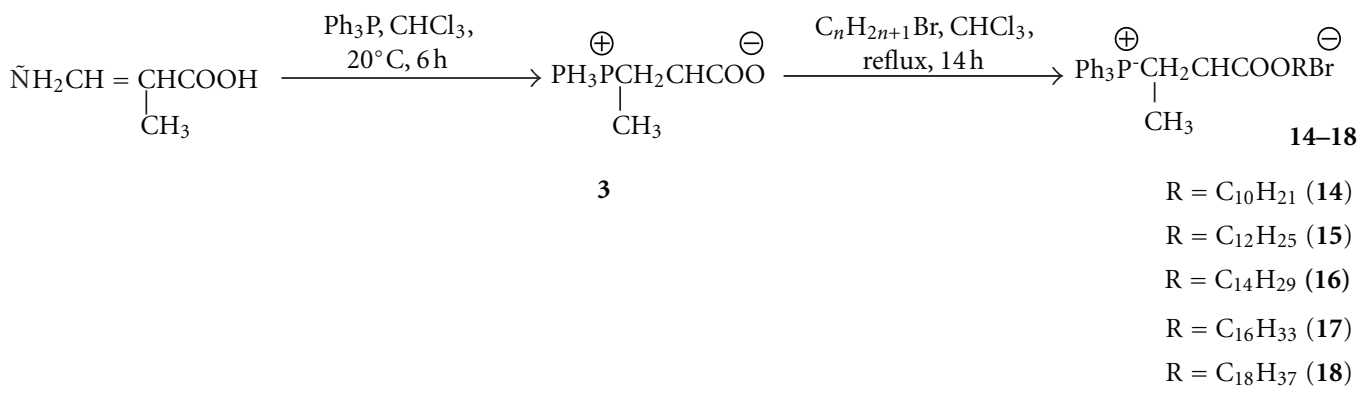

Scheme 4: Synthetic routes of $\beta$-(carboxyalkyl)- $\alpha$-methylethyltriphenylphosphonium bromides 14-18; reagents and conditions.

Griseofulvin (Sigma-Aldrich) was used as reference drug for fungi. Every experiment in the antibacterial and antifungal assay was replicated twice. For the antibacterial and antifungal activity, the compounds were dissolved in dimethylsulfoxide (DMSO).

Many years we thought that it is impossible to grow single crystals of oil products 4-18 with long alkyl chains suitable for X-ray diffraction, but after five years we have a real chance to obtain the crystalline structure of the $\mathrm{Ph}_{3} \mathrm{PCH}_{2} \mathrm{CH}_{2} \mathrm{C}(\mathrm{O}) \mathrm{OC}_{16} \mathrm{H}_{33}$ (7), which gave good quality crystals (Figure 1) [11].

2.5.1. Antibacterial Activity. Different strains of bacteria were used as Staphylococcus aureus (ATCC 29213), Escherichia coli (ATCC 25922), Pseudomonas aeruginosa (ATCC 27853), and Proteus mirabilis (ATCC 12453). Cup-plate Agar method was used for evaluation of antibacterial activity. The nutrient agar medium is used. The medium with bacteria was poured into sterilized Petri dishes under aseptic conditions. Standard drugs used were Penicillin $(50 \mu \mathrm{g} / 0.1 \mathrm{~mL})$ and Chlorhexidine $(50 \mu \mathrm{g} / 0.1 \mathrm{~mL})$ and test compounds at concentration of $50 \mu \mathrm{g} / 0.1 \mathrm{~mL}$. Solvent used was dimethyl sulfoxide (DMSO). Plates were incubated at $37^{\circ} \mathrm{C}$ for 24 hours. After incubation the average zone of inhibition was recorded in $\mathrm{mm}$ [12].

2.5.2. Antifungal Activity. The antifungal activity was carried out by using cup-plate method using Sabouraud's agar medium. Fungal strains used were Candida albicans (ATCC 885-653) with incubation period of 48 hours at temperature $28^{\circ} \mathrm{C}$. The standard drug used was Griseofulvin
$(50 \mu \mathrm{g} / 0.1 \mathrm{~mL})$ and the test compounds at concentration of $50 \mu \mathrm{g} / 0.1 \mathrm{~mL}$ by using dimethyl sulfoxide (DMSO) [13].

\section{Results and Discussions}

3.1. Chemistry. The synthetic routes are given in Schemes $2-4$. In this paper we present the synthesis and biological activity of a series of nanosized $(30 \mathrm{~nm})$ quaternary phosphonium salts 4-18 with long alkyl chains $\left(\mathrm{R}=\mathrm{C}_{n} \mathrm{H}_{2 n+1} ; n=\right.$ $10,12,14,16,18$; here $n$ is the number of carbon atoms in alkyl groups) on the basis of phosphabetaines 1-3 and higher alkyl halogenides. All the synthesized compounds were characterized by elemental analysis, IR, ${ }^{1} \mathrm{H} N M R$, and ${ }^{31} \mathrm{P}$ NMR spectroscopy. A crystalline product of $\beta$ (carboxyhexadecyl)ethyltriphenylphosphonium bromide 7 was prepared and characterized by single-crystal X-ray analysis [11] (Figure 1).

3.1.1. Scheme 2 Depicts the Synthesis of $\beta$-(Carboxyalkyl) ethyltriphenylphosphonium Bromides (4-8). Treatment of acrylic acid with triphenylphosphine at room temperature in chloroform during 6 hours yielded (78\%) phosphabetaine 1. Alkylation of the starting phosphabetaine 1$\beta$-triphenylphosphonium ethylcarboxylate with alkyl halogenides (reflux for two hours in $\mathrm{CH}_{3} \mathrm{Cl}$ ) gave the corresponding phosphonium bromides 4-8 with long alkyl chains. Molecular structure of product 7 is given in Figure 1.

3.1.2. Scheme 3 Depicts the Synthesis of $\beta$-(Carboxyalkyl)$\beta$-methylethyltriphenylphosphonium Bromides 9-13. Treatment of crotonic acid with triphenylphosphine at room 
TABLE 1: Antimicrobial activity of the newly synthesized compounds and the control drugs $(50 \mu \mathrm{g} / 0.1 \mathrm{~mL})$.

\begin{tabular}{|c|c|c|c|c|c|c|}
\hline \multirow[b]{2}{*}{$n$} & \multirow[b]{2}{*}{ Compound } & \multicolumn{5}{|c|}{ Zone of inhibition (mm) } \\
\hline & & $\begin{array}{l}\text { Staphylococcus } \\
\text { aureus }\end{array}$ & Escherichia coli & $\begin{array}{l}\text { Pseudomonas } \\
\text { aeruginosa }\end{array}$ & Proteus mirabilis & $\begin{array}{l}\text { Candida } \\
\text { albicans }\end{array}$ \\
\hline 4 & $\mathrm{Ph}_{3} \stackrel{\oplus}{\mathrm{P}}-\mathrm{CH}_{2} \mathrm{CH}_{2} \mathrm{C}(\mathrm{O}) \mathrm{ON}_{10} \mathrm{H}_{21} \stackrel{\ominus}{\mathrm{Br}}$ & 15 & 20 & 21 & 18 & 24 \\
\hline 5 & $\mathrm{Ph}_{3} \stackrel{\oplus}{\mathrm{P}}-\mathrm{CH}_{2} \mathrm{CH}_{2} \mathrm{C}(\mathrm{O}) \mathrm{ON}_{12} \mathrm{H}_{25} \stackrel{\ominus}{\mathrm{Br}}$ & 20 & 22 & 13.5 & 23 & 28 \\
\hline 6 & $\mathrm{Ph}_{3} \mathrm{P}-\mathrm{CH}_{2} \mathrm{CH}_{2} \mathrm{C}(\mathrm{O}) \mathrm{ON}_{14} \mathrm{H}_{29} \stackrel{\ominus}{\mathrm{Br}}$ & 25 & 17 & 14 & 10 & 24 \\
\hline 7 & $\mathrm{Ph}_{3} \stackrel{\oplus}{\mathrm{P}}-\mathrm{CH}_{2} \mathrm{CH}_{2} \mathrm{C}(\mathrm{O}) \mathrm{O}_{16} \mathrm{H}_{33} \stackrel{\ominus}{\mathrm{Br}}$ & 25 & 18 & 11 & 17 & 22 \\
\hline 8 & $\mathrm{Ph}_{3} \stackrel{\oplus}{\mathrm{P}}-\mathrm{CH}_{2} \mathrm{CH}_{2} \mathrm{C}(\mathrm{O}) \mathrm{O} \tilde{\mathrm{N}}_{18} \mathrm{H}_{37} \stackrel{\ominus}{\mathrm{Br}}$ & 23 & 14 & 13 & 8 & 24 \\
\hline 9 & $\mathrm{Ph}_{3} \stackrel{\oplus}{\mathrm{P}}-\underset{\mid}{\mathrm{CH}_{3}} \mathrm{CHCH}_{2} \mathrm{C}(\mathrm{O}) \mathrm{OC}_{10} \mathrm{H}_{21} \stackrel{\ominus}{\mathrm{Br}}$ & 17 & 10 & 17 & 16 & 23 \\
\hline 10 & 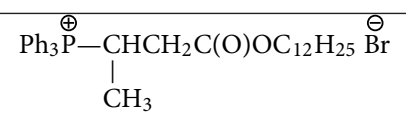 & 21 & 17 & 15 & 11 & 20.5 \\
\hline 11 & $\mathrm{Ph}_{3} \stackrel{\oplus}{\mathrm{P}}-\underset{\mid}{\mathrm{C}} \mathrm{CHCH}_{2} \mathrm{C}(\mathrm{O}) \mathrm{OC}_{14} \mathrm{H}_{29} \stackrel{\ominus}{\mathrm{Br}}$ & 26 & 17 & 14 & 13 & 25 \\
\hline 12 & $\mathrm{Ph}_{3} \stackrel{\oplus}{\mathrm{P}}-\underset{{ }_{\mathrm{CH}}}{\mathrm{CHCH}_{2} \mathrm{C}(\mathrm{O}) \mathrm{OC}_{16} \mathrm{H}_{33} \mathrm{Br}} \stackrel{\ominus}{\mathrm{Br}}$ & 20 & 14 & 15 & 11 & 21 \\
\hline 13 & $\mathrm{Ph}_{3} \stackrel{\oplus}{\mathrm{P}}-\underset{\text { l }}{\mathrm{C}} \mathrm{CH}_{3} \mathrm{HCH}_{2} \mathrm{C}(\mathrm{O}) \mathrm{OC}_{18} \mathrm{H}_{37} \stackrel{\ominus}{\mathrm{Br}}$ & 19 & 13 & 10.5 & 9 & 20 \\
\hline 14 & $\mathrm{Ph}_{3} \mathrm{P}-\mathrm{\oplus}_{\mid} \mathrm{CH}_{2} \mathrm{CHC}(\mathrm{O}) \mathrm{OC}_{10} \mathrm{H}_{21} \stackrel{\ominus}{\mathrm{Br}}$ & 17 & 14 & 15 & 11 & 21.5 \\
\hline 15 & $\mathrm{Ph}_{3} \mathrm{P}-\mathrm{CH}_{2} \mathrm{CHC}(\mathrm{O}) \mathrm{OC}_{12} \mathrm{H}_{25} \stackrel{\ominus}{\mathrm{Br}}$ & 25.5 & 17 & 18 & 15 & 25 \\
\hline 16 & $\underset{\mathrm{Ch}_{3} \mathrm{P}-\mathrm{CH}_{2} \mathrm{CHC}(\mathrm{O}) \mathrm{OC}_{14} \mathrm{H}_{29} \stackrel{\ominus}{\mathrm{Br}}}{\mid}$ & 25 & 19 & 17.5 & 17 & 28 \\
\hline 17 & $\begin{array}{c}\mathrm{Ph}_{3} \mathrm{P}-\mathrm{CH}_{2} \mathrm{CHC}(\mathrm{O}) \mathrm{OC}_{16} \mathrm{H}_{33} \stackrel{\ominus}{\mathrm{Br}} \\
\stackrel{\mid}{\mathrm{C}} \mathrm{H}_{3}\end{array}$ & 17 & 13.5 & 14 & 11.5 & 20 \\
\hline 18 & $\begin{array}{c}\mathrm{Ph}_{3} \mathrm{P}-\mathrm{CH}_{2} \mathrm{CHC}(\mathrm{O}) \mathrm{OC}_{18} \mathrm{H}_{37} \stackrel{\ominus}{\mathrm{Br}} \\
\stackrel{\mathrm{CH}}{\mid}\end{array}$ & 19 & 13 & 11 & 17 & 19 \\
\hline 19 & Chlorhexidine & 16 & 15 & 13 & 14 & 16.5 \\
\hline 20 & Penicillin & 23 & 16 & 8 & 10 & - \\
\hline 21 & Griseofulvin & - & - & - & - & 19 \\
\hline
\end{tabular}

$n$ : Compound number.

All tests were performed in triplicate. Zone of inhibition 20 to 28: highly significant, between 11 and $19 \mathrm{~mm}$ : less significant, below $10 \mathrm{~mm}$ : poor active.

temperature in chloroform during 8 hours yielded (75\%) phosphabetaine 2 . Alkylation of the starting phosphabetaine 2 - $\beta$-triphenylphosphonium $\beta$-methylethylcarboxylate with alkyl halogenides (reflux for 10 hours in $\mathrm{CH}_{3} \mathrm{Cl}$ ) gave the corresponding phosphonium bromides 9-13 with long alkyl chains. The yield was $60 \%-70 \%$.
3.1.3. Scheme 4 Depicts the Synthesis of $\beta$-(Carboxyalkyl)- $\alpha$ methylethyltriphenylphosphonium Bromides (14-18). Treatment of methacrylic acid with triphenylphosphine at room temperature in chloroform during 6 hours yielded (77\%) phosphabetaine 3. Alkylation of the starting phosphabetaine 3- $\beta$-triphenylphosphonium- $\alpha$-methylethylcarboxylate with 
alkyl halogenides (reflux for 14 hours in $\mathrm{CH}_{3} \mathrm{Cl}$ ) gave the corresponding phosphonium bromides 14-18 with long alkyl chains. The yield was $70 \%-90 \%$.

3.2. Antimicrobial Activity. The synthesized compounds-a new class of bioactive nanomolecules $(30 \mathrm{~nm})$-were screened for antibacterial and antifungal activity at $50 \mu \mathrm{g} /$ $0.1 \mathrm{~mL}$ concentration by using the cup-plate agar diffusion method, and standard drugs used were Chlorhexidine 19, Penicillin 20, and Griseofulvin 21. The novel synthesized compounds 4-18 with long alkyl chains $(n=10,12,14$, 16 , and 18) show maximal activity against pathogenic microorganisms. Starting phosphabetaines 1-3 and all phosphonium salts with short alkyl chains, synthesized earlier [6], were not active at all. Compounds 4, 5, 6, 7, 11, $\mathbf{1 5}$, and 16 were highly significant against tested bacteria as well as fungi. Our results are reported in Table 1.

Such a high biological activity of cationic biocides 4-18 we explain by their ability to be integrated into the lipid layers of biomembranes of pathogenic microflora eventually leading to the destruction of this last [14]. To confirm this idea we studied the interaction mechanism of compounds 4-8 - synthetic phosphorus analogs of biomembranes-with natural biological membranes (lecithin) using the model of Langmuir monolayers [15]. It was discovered that alkylated phosphabetaines 4-8 interact with lecithin, by forming a pores, and thus deteriorating the membrane functions.

\section{Conclusion}

In conclusion, carboxylate phosphabetaines derivatives with alkyl chains of various lengths were synthesized in good yield, characterized by different spectral studies, and their antimicrobial activity has been evaluated. Compounds 5-8, $\mathbf{1 1}, \mathbf{1 5}$, and $\mathbf{1 6}$ demonstrated good inhibitions against all the strains tested comparable to Chlorhexidine, Penicillin, and Griseofulvin as positive standard. So, it may be concluded from our results that the synthesized compounds are potent nanoantimicrobial agents against pathogenic bacteria and fungi.

\section{Acknowledgment}

The authors thank Academy of Sciences of Tatarstan Republic, Russia, for financial support of this research work.

\section{References}

[1] G. Aysel, I. Taylan, T. Nalan, and O. Gulten, "Synthesis and antimicrobial evaluayion of some novel imidazolylmercaptjacetylthio semicarbazide and 4-yhiazolidinone analogs," Turkish Journal of Pharmaceutical Sciences, vol. 2, no. 1, pp. 134-136, 2005.

[2] J. W. Costerton and K.-J. Cheng, "The role of the bacterial cell envelope in antibiotic resistance," Journal of Antimicrobial Chemotherapy, vol. 1, no. 4, pp. 363-377, 1975.

[3] H. Kourai, T. Horie, and K. Takeichi, "The antimicrobial characteristics of quaternary ammonium salts and their alkyl chain length," Journal of Antibacterial and Antifungal Agents, vol. 8, pp. 9-17, 1980.

[4] A. Kanazawa, T. Ikeda, and T. Endo, "Synthesis and antimicrobial activity of dimethyl- and trimethyl-substituted phosphonium salts with alkyl chains of various lengths," Antimicrobial Agents and Chemotherapy, vol. 38, no. 5, pp. 945-952, 1994.

[5] V. I. Galkin, Y. V. Bakhtiyarova, N. A. Polezhaeva, R. A. Shaikhutdinov, and V. V. Klochkov, "Synthesis and properties of phosphabetainic structures. Triphenylphosphine and tpiphenylphosphite in reactions with unsaturated carboxylic acids and their derivatives," Zhurnal Obshchei Khimii, vol. 68, pp. 1104-1108, 1998.

[6] V. I. Galkin, Y. V. Bakhtiyarova, N. A. Polezhaeva et al., "Synthesis, structure and reactivity of carboxylate phosphabetaines," Phosphorus, Sulfur, and Silicon and the Related Elements, vol. 144-146, pp. 918-920, 1999.

[7] V. I. Galkin, Y. V. Bakhtiyarova, A. N. Zaripova et al., "Phosphabetaines on the basis of triphenylphosphine and unsaturated dicarboxylic acids," Phosphorus, Sulfur and Silicon and Related Elements, vol. 177, no. 8-9, pp. 2063-2065, 2002.

[8] V. I. Galkin, Y. V. Bakhtiyarova, R. I. Sagdieva, I. V. Galkina, and R. A. Cherkasov, "The synthesis and reactions of betaines formed in reactions of tertiary phosphines with unsaturated carboxylic acids and their derivatives," Heteroatom Chemistry, vol. 17, no. 6, pp. 557-566, 2006.

[9] P. R. Murray and J. R. Zeitinger, "Evaluation of Mueller-Hinten agar for disk diffusion susceptibility tests," Journal of Clinical Microbiology, vol. 18, no. 5, pp. 1269-1271, 1983.

[10] A. W. Bauer, W. M. M. Kirby, J. C. Sherris, and M. Turck, "Antibiotic susceptibility testing by a standardized single disk method," American Journal of Clinical Pathology, vol. 45, no. 4, pp. 493-496, 1966.

[11] G. A. Gainanova, I. G. Vagapova, V. V. Syakaev et al., "Selfassembling systems based on amphiphilic alkyltriphenylphosphonium bromides: elucidation of the role of head group," Journal of Colloid and Interface Science, vol. 367, no. 1, pp. 327-336, 2012.

[12] J. M. Andrews, "Determination of minimum inhibitory concentrations," Journal of Antimicrobial Chemotherapy, vol. 48, supplement 1, pp. 5-16, 2001.

[13] J. P. Phair, C. Watanakunakorn, and T. Bannister, "In vitro susceptibility of Pseudomonas aeruginosa to carbenicillin and the combination of carbenicillin and gentamicin," Applied Microbiology, vol. 18, no. 3, pp. 303-306, 1969.

[14] N. Togashi, A. Shiraishi, M. Nishizaka et al., "Antibacterial activity of long-chain fatty alcohols against Staphylococcus aureus," Molecules, vol. 12, no. 2, pp. 139-148, 2007.

[15] I. V. Galkina, N. B. Melnikova, Y. V. Bakhtiyarova et al., "Interaction of alkylated phosphabetaines with biomembranes lipid compounents," Pharmaciya, vol. 3, pp. 47-49, 2010. 

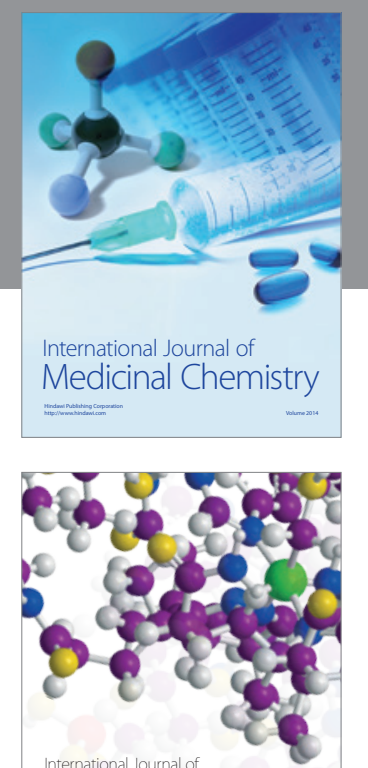

\section{Carbohydrate} Chemistry

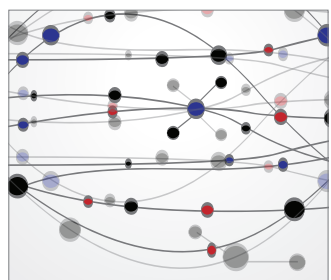

The Scientific World Journal
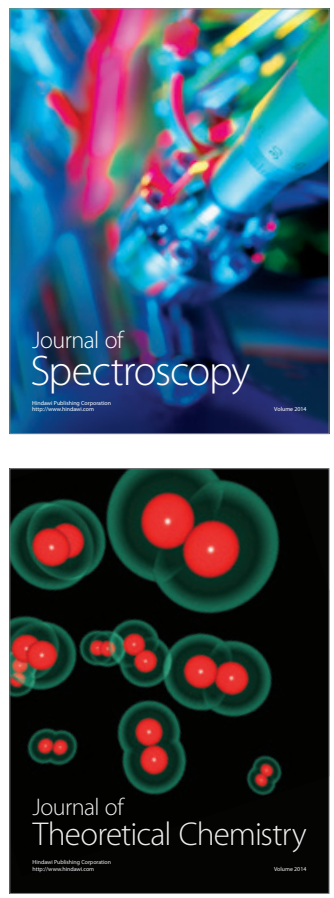
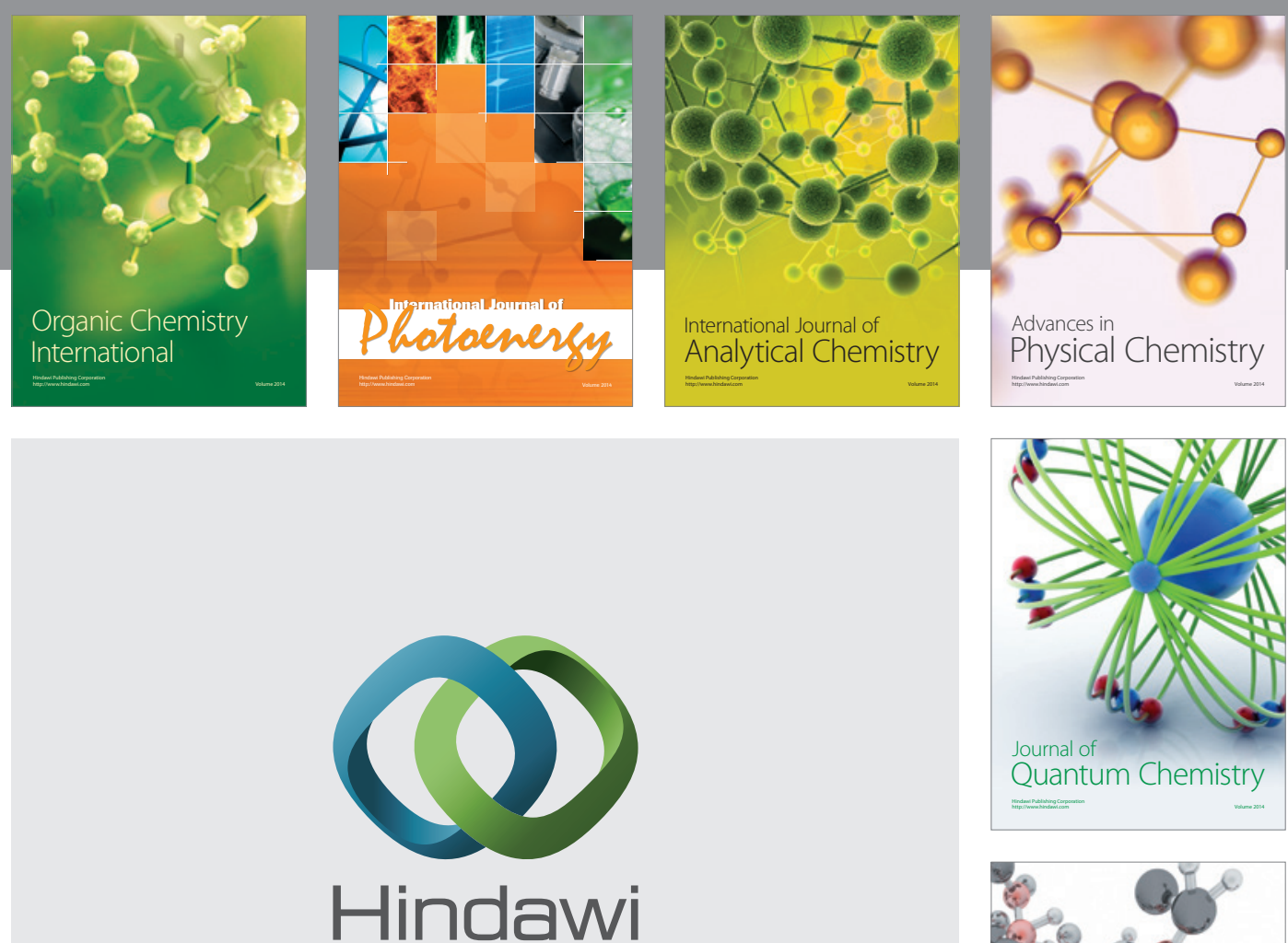

Submit your manuscripts at

http://www.hindawi.com

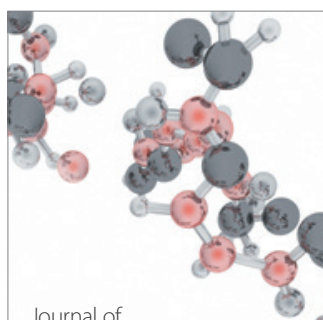

Analytical Methods

in Chemistry

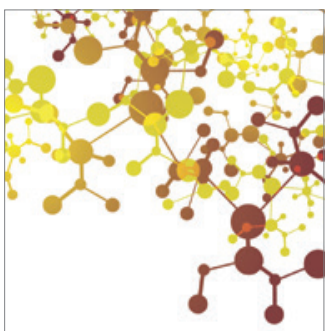

Journal of

Applied Chemistry

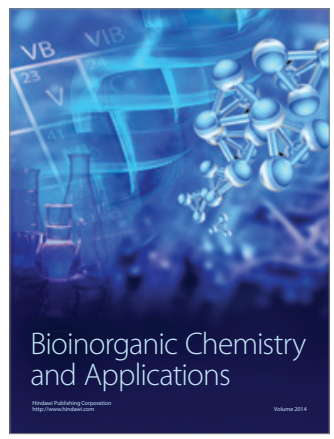

Inorganic Chemistry
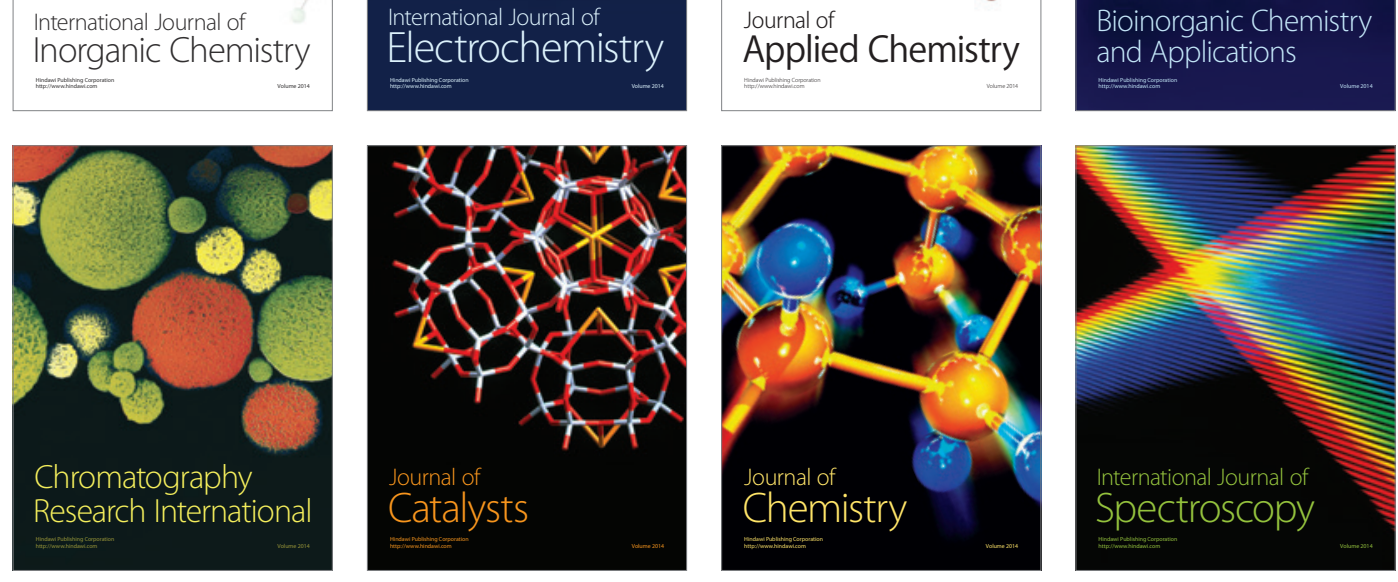